Militello, G., Bich, L. \& Moreno, A. (2020). Functional integration and individuality in prokaryotic collective organisations. Acta Biotheoretica, doi: 10.1007/s10441-020-09390-z

\title{
Functional integration and individuality in prokaryotic collective organisations
}

\author{
Guglielmo Militello \\ ORCID: 0000-0003-2657-3977 \\ IAS-Research Centre for Life, Mind and Society. Department of Logic and Philosophy of Science. University of the \\ Basque Country (UPV/EHU). Avenida de Tolosa 70, Donostia-San Sebastian, 20018, Spain.
}

Leonardo Bich

ORCID: 0000-0002-2416-112X

IAS-Research Centre for Life, Mind and Society. Department of Logic and Philosophy of Science. University of the Basque Country (UPV/EHU). Avenida de Tolosa 70, Donostia-San Sebastian, 20018, Spain.

*Corresponding author: leonardo.bich@ehu.es

Alvaro Moreno

ORCID: 0000-0002-2612-8403

IAS-Research Centre for Life, Mind and Society. Department of Logic and Philosophy of Science. University of the Basque Country (UPV/EHU). Avenida de Tolosa 70, Donostia-San Sebastian, 20018, Spain.

\begin{abstract}
Both physiological and evolutionary criteria of biological individuality are underpinned by the idea that an individual is a functionally integrated whole. However, a precise account of functional integration has not been provided so far, and current notions are not developed in the details, especially in the case of composite systems. To address this issue, this paper focuses on the organisational dimension of two representative associations of prokaryotes: biofilms and the endosymbiosis between prokaryotes. Some critical voices have been raised against the thesis that biofilms are biological individuals. Nevertheless, it has not been investigated which structural and functional obstacles may prevent them from being fully integrated physiological or evolutionary units. By contrast, the endosymbiotic association of different species of prokaryotes has the potential for achieving a different type of physiological integration based on a common boundary and interlocked functions. This type of association had made it possible, under specific conditions, to evolve endosymbionts into fully integrated organelles. This paper therefore has three aims: first, to analyse the organisational conditions and the physiological mechanisms that enable integration in prokaryotic associations; second, to discuss the organisational differences between biofilms and prokaryotic endosymbiosis and the types of integration they achieve; finally, to provide a more precise account of functional integration based on these case studies.
\end{abstract}

Keywords: Biofilm; Control; Endosymbiosis; Engulfment; Eukaryogenesis; Regulation. 


\section{Introduction}

Collective associations are widespread in the biological world and give rise to very different organisations ranging from associations of bacteria and archaea to societies of multicellular organisms (e.g., social insects). However, only in certain cases these associations become an integrated individual. Thus, an intense debate about when collective associations constitute a new individuality has been taking place during the last decades. The central question underlying this debate can be summarised with Wilson's words: "at what point does a society become so well integrated that it is no longer a society" (Wilson 1974, p. 54). Although Wilson's question refers to animal societies, it is so general that it can apply to any kind of biological association. Indeed, it clearly emphasises three fundamental aspects of the transition from a biological association to an individual: first, there are some specific conditions (summarised by "at what point") that permit an association to become a more cohesive whole; secondly, the transformation of an association into a more cohesive whole involves the achievement of a certain degree of (functional) integration among the constituent organisms (indeed, they must be "well integrated"); finally, the process of integration among the parts of the association leads to something "that is no longer a society", therefore a (new) individual (i.e. an organism-like associative entity).

In the contemporary debate about composite biological individuality (e.g., biofilms, holobionts, colonies of insects), the two main approaches usually adopted - i.e. evolutionary and physiological - rely on the idea that a biological individual is an integrated whole whose functions are strongly interconnected. Yet little has been said about the conditions that may enable an association to become a functionally integrated individual and what mechanisms are involved. The reason lies in the fact that the very concept of functional integration, often considered as a synonym for 'physiological integration' (Pradeu, 2010), has not been characterised in detail. To further complicate matters, functional integration is an especially multifaceted and complex aspect of biological organisation that includes several important dimensions of a biological system such as metabolic, regulatory and sensorimotor abilities, development, immunological responses, reproduction, etc. As a consequence of the unclear character of the notion of integration, not only current general definitions of biological individuality that appeal to it are somehow undermined, but also the mechanisms allowing an association to become a more integrated whole are mostly unexplored.

In an attempt to develop the notion of integration in more detail with the help of biological examples, we first analyse the fundamental physiological mechanisms that could explain the transition from an association of bacteria towards a new full-fledged, functionally integrated individuality; second, we examine the different types and degrees of functional integration enabled by different mechanisms, by taking into consideration their limits and potentials (understood as enabling conditions) to bring about further forms of integration. In particular, by adopting an organisational approach, we aim to connect the physiological dimension of the process of individuation with the evolutionary one through an analysis of the role of the different forms of physiological integration in the reproduction of a collective entity. Finally, we provide a more precise characterisation of the notion of 'functional integration'. It is worth pointing out that this paper is not aimed at drawing up a list of properties (or criteria) that sharply distinguish a loose association of organisms from an individual, but rather at exploring the conditions that can potentially permit the transition from the former to the latter and, on this basis, at contributing to a better understanding of what a 'functionally integrated individual' is.

Despite the great variety of biological collective associations, we have chosen to focus on two case-studies from the bacterial and archaeal domains: biofilms (i.e. colonies of single- and multispecies bacteria and archaea) and the endosymbiotic relationship between two species of bacteria ${ }^{1}$. These case-studies have been

\footnotetext{
1 Other collective associations of prokaryotes include colonies stemming from the clones of single species bacteria/archaea (e.g. Lactococcus lactis or Streptococcus thermophylus) or intracellular parasites (e.g. Vampirococcus and Bdellovibrio). We will not analyse these cases here as they do not exhibit the features of a stable functionally
} 
chosen for three reasons: first, they are minimal forms of composite biological systems; second, they exhibit different physiological mechanisms that allow understanding why the collective association achieves in each case a very different degree of functional integration among its parts; finally, the endosymbiotic relationship between two species of bacteria may provide important clues as to the origin of a paradigmatic example of a new functionally integrated individual, the eukaryotic cell ${ }^{2}$.

The connection between our case-studies lies in three main aspects: first, a common spatial constraint (i.e. the ECM and the membrane of Tremblaya) that surrounds a set of prokaryotic cells; secondly, the systemic control of and among the parts, enabled by the common boundary, which affects the type and degree of physiological integration achieved by the association; thirdly, the evolutionary potential opened up by different kinds of common boundaries. As we shall argue in the detail, biofilms and the endosymbiosis between bacteria have diverse spatial organisations that constrain their constituting organisms differently, providing different mechanisms of collective control ${ }^{3}$. This provides them with a distinct type of stability over time and opens up different evolutionary possibilities for an association to give rise to an integrated individual. This thesis has two important explanatory consequences: first, it sheds light on the connection between spatial constraints and physiological mechanisms for explaining the functional integration of collective associations; secondly, it clarifies some important physiological dimensions of the idea of 'functional integration', thereby helping to better define the meaning of a 'functionally integrated individuality'.

The paper is structured as follows. Section 2 examines some philosophical accounts that appeal to the notion of functional integration to ascribe individuality to biological associations. Section 3 analyses the organisation of biofilms and discusses the role of the extracellular polymeric substance (EPS) matrix in constraining and integrating the activity of the prokaryotic cells that compose them. Section 4 examines the organisational role played by engulfment and cross-control in the Tremblaya-Moranella association, the only well-studied case of endosymbiotic relationship between prokaryotes ${ }^{4}$. Section 5 discusses the organisational differences between these two forms of association (biofilms and endosymbiosis) by focusing on the roles of the EPS matrix and engulfment. Hence, it examines the main organisational issues raised by endosymbiosis and how they may have been solved during eukaryogenesis. Finally, the last section draws some conclusions on functional integration and biological individuality.

\section{Association and integration in biological collective organisations: a critical review}

The concept of 'functional integration' is often invoked in the debate on biological individuality as a necessary element in developing an understanding of how living systems constitute coherent wholes both in evolution and physiology. In this context, this concept is usually employed as an explanans, rather than the main object of investigation, as the aim is to build general accounts of individuality. In the context of the debate on evolutionary individuality, for example, Hull (1980) pointed out that in order to be an object of natural selection a biological system must be able not only to undergo genetic variation and transmit it to the

integrated collective organisation. In the first case, they do not exhibit a common spatial constraint, the EPS matrix. In the second case intracellular parasitism is a transient not functionally integrated relationship where the host is killed.

2 The focus on current forms of endosymbiosis as a possible way to provide valuable clues as to the role played by endosymbiosis in the achievement of a 'strong' physiological integration in eukaryogenesis moves in a similar direction to the one explored by Reyes-Prieto et al. (2014). They focus on non-autonomous endosymbionts with extremely reduced genomes, or 'symbionelles', to shed light on the origin of eukaryotic organelles.

${ }^{3}$ This idea is in line with the thesis that all multicellular association need to solve the issue of spatial control, and that different ways of doing so result in different types of organisations and different degrees of integration (Bich et al. 2019).

${ }^{4}$ Although the term 'prokaryote' is nowadays substituted by Bacteria and Archaea, for the sake of simplicity we continue to employ this word in the paper to intend the unicellular organisms belonging to the two domains of Bacteria and Archaea. 
offspring, but also to interact with the environment as a cohesive physiological whole. Moreover, integration underlies reproductive capabilities. As Sober (1991) observes, all groups (e.g. colonies of insects, groups of cells, parasitic relationships) exhibit a certain kind of functional interdependence which consists of "parts of different sorts and these parts interact so as to sustain the organism and allow it to reproduce" (p. 275). Sober suggests that all those functions involved in self-maintenance (e.g. metabolism) and reproduction somehow exhibit interdependence. Integration is also used to account for the absence of conflict and the presence of a high cooperativity among the component parts in such a way that they work as "bundles of adaptation", where all elements work toward a common evolutionary goal (Queller and Strassman 2009, 2016). In the same vein, Dupré and O'Malley (2009) emphasise that the functional integration of living beings, including collective associations, is characterised by the interconnection of metabolic pathways and reproduction. Metabolic processes are described generically as collaborative activities that entail a certain degree of functional interdependence. In the case of symbiotic associations, this usually takes the form of cometabolism and synthrophy. Reproduction is a more complex issue in collective associations because vertical transmission and parent-offspring lineages do not always occur (See Skillings, 2016).

It is important to point out that the fundamental aim of this debate is to address the notion of individuality and not so much to clarify in the detail which types of mechanisms are required for self-maintenance, reproduction and cooperation. Functional integration, therefore, is used as a general notion in this context.

Hence, biological individuality understood in terms of physiology has received much less attention in the literature than evolutionary accounts. In this domain the general notion of functional integration is expected to play an even more important role. In fact, it is still employed generically. It has been emphasised, for example, that the immune system plays a key role in explaining the interdependence of the functional parts of organisms and collective associations, because immune interactions "are systemic (as opposed to local) and [...] responsible for the acceptance or rejection of constituents in the organism" (Pradeu 2010, p. 258; see also Howes, 1998). This view, however, does not take into consideration the complexity of biological integration and the fact that a systemic control is performed not only by the immune system, but also by other regulatory subsystems and mechanisms that modulate and coordinate the functions of the components of the system. Moreover, the immune system depends on and is maintained by a more comprehensive physiological regime, which provides the energy for its functioning and coordinates immune activity with those of the other functional subsystems.

Whereas the philosophical approaches mentioned above rely on the notion of integration as a generic concept (mostly as an explanans) to develop general accounts of individuality, this paper aims to make this notion the main focus of the paper and to address it as an explanandum. The objective is to analyse in the details how functional integration is achieved in biological associations by focusing on specific case studies. It is important to clarify two points. The first concerns the approach adopted. The paper focuses on the organisational aspects underlying integration in biological associations, with the aim to identify what mechanisms enable transitions from loose collective associations to cohesive physiological wholes that are also capable to reproduce and evolve as units of selection. Focusing on current organisations (Moreno and Mossio 2015) means in the first place identifying the physiological mechanisms that make possible different degrees and types of integration. Yet it also implies identifying the possibilities and bottlenecks of different types of organisation on the evolutionary scale.

The second point is the choice of the case study. To address the problem of how functional integration is achieved by biological associations and to explore how it can lead to a fully integrated individual, we focus on associations of prokaryotes (Bacteria and Archaea). The advantages are two. It is a minimal case, whose organisational features are expected to be less complex than in associations of eukaryotic cells, colonies of insects etc. In addition, it is widely accepted that it was from associations of prokaryotes that the eukaryotic cell originated. Therefore, the eukaryotic cell, which is widely accepted as a case of full-fledged biological individual, can work as a term of reference to discuss how far functional integration can in principle develop 
from the association of different prokaryotic organisms. This strategy allows us to identify how distinct mechanisms can lead to different types and degree of integration.

Several works have emphasised the importance of control and regulatory mechanisms in realising integration in different types of associations, from the development of multicellular systems (Arnellos et al. 2014; Griesemer 2016, Bich et al. 2019) to the physiology of symbiotic relationships (Catania et al. 2017; Bich 2019) ${ }^{5}$. Queller and Strassmann $(2009,2016)$, for example, have called into question the importance of spatial contiguity, the indivisibility of the parts, the development from a single lineage and the genetic uniformity among the members, to point out that it is the control of conflict and a high cooperation among the members of a society that are necessary for achieving a sufficient degree of functional integration. It is worth noting that most of these accounts have focused on associations of eukaryotic organisms which exhibit forms and degrees of integration that are different from those of the prokaryotic world. Indeed, whereas the former ones give rise to multicellular integrated individuals, it is doubtful whether also the latter do so.

The discussion of these ideas in the specific context of prokaryotic associations has generated a debate regarding the status of biofilms; in particular, whether or not (and why) they can be considered integrated individuals. Ereshefsky and Pedroso (2013, 2015) and Doolittle (2013) have recently argued that multispecies biofilms can be considered individuals, because their extracellular matrix allows for a unitary interaction with their environment and because they are capable of reproduction, although they lack a high degree of germ-soma specialisation. In response, Clarke (2016) has argued that a biofilm does not actually interact as a whole, because "most interactions take place across spatial scales that are much smaller than an entire biofilm" (p. 205). In addition, since they do not have a collective reproductive system and bacteria can enter or exit the biofilm, biofilms cannot reproduce as wholes and they cannot vertically transmit genetic variations to future generations. Therefore, they cannot undergo group selection (Clarke 2016). According to Clarke (2016), in spite of exhibiting a certain degree of functional cohesion resulting from metabolic codependence and a certain form of collective border, biofilms do not perform collective mechanisms of interaction and reproduction, and therefore they do not evolve as individuals. One may suspect that these features depend on the fact that the bacterial components still maintain a sufficiently high degree of autonomy and the biofilm as a system lacks more comprehensive (global) ways to control the behaviour of the bacteria.

To explore this different hypothesis, we will analyse the organisational role played by the extracellular polymeric substance (EPS) matrix and other control mechanisms in biofilms. The aim is to understand how such structures and mechanisms enable a certain type and degree of functional integration in biofilms, and to compare it with another type of organisation deriving from the endosymbiosis between bacteria, which has the potential for a different and stronger type of integration.

\section{Collective integration in biofilms: distributed control and the role of the EPS matrix}

Biofilms are biological systems realised by ecological communities of (single- or multispecies) bacteria and archaea and by the extracellular polymeric matrix they produce. The development of a biofilm includes three sequential steps: first, the attachment of bacteria (or archaea) to a surface and the formation of a monolayer structure (that binds the bacteria together and to the surface); second, cell division and the production and deposition of the EPS matrix, which gives rise to a multilayer organisation; third, the disassembly of the matrix and the dispersion of cells..

\footnotetext{
${ }^{5}$ As pointed out by Catania et al. (2017), regulatory networks play a pivotal role in defining the functional integration of symbiotic partners. These interdependent networks may be co-inherited (via vertical gene transfer) or re-established in a new generation (via horizontal gene transfer). This argument is also in line with Bich et al. (2016), who have argued that a functionally integrated organisation hinges on a complex set of regulatory mechanisms that allow it to coordinate the contributions of its functional parts and to handle perturbations.
} 
In the first stage of biofilm's life cycle, individual cells attach to a biotic or abiotic surface by means of $a_{\text {adhesins }}^{6}$ and give rise to a monolayer biofilm (Karatan and Watnick 2009). The production of adhesins is triggered by the concentration of specific substances (e.g. oxygen or sugars) in the environment. The second stage begins when the spatial proximity of cells triggers the emission of several extracellular signals (e.g. mechanical, metabolic, inorganic, etc.) and the activation of quorum-sensing (QS) mechanisms, that collectively promote the synthesis and deposition of extracellular matrix components. At this stage, cells may attach to one another and to the EPS matrix, thus realising a multilayer biofilm where they undergo proliferation and differentiation into several cell types (Lopez et al. 2009). In the third stage, the EPS matrix disassembles and causes biofilm dispersion. This occurs in presence of a massive accumulation of toxic waste products, or when the system grows beyond the transport and distribution capabilities of EPS channels and the innermost layers of cells cannot receive enough nutrients. Biofilms employ several regulatory mechanisms that trigger dispersion in response to different stimuli (e.g. variations in concentrations of nutritional cues, oxygen and nitric oxide, presence of death bacteria) (Karatan and Watnik 2009).

Thanks to (at least) three types of extra- and inter-cellular control mechanisms - QS, EPS matrix and bacterial conjugation - a biofilm becomes a cohesive functional unit whose parts act and are maintained together. Bacterial conjugation is somehow favoured by the close spatial proximity of cells in specific areas of the biofilm, but it works locally at short (cell-to-cell) ranges. Therefore, we will focus on the former two, which act at medium ranges ${ }^{7}$. They constitute the main factors of integration of the whole system, because they are responsible for the overall development and functioning of the biofilm. QS is a distributed control system that relies on the concentrations of a set of signalling molecules that allow bacteria to coordinate their gene expression and trigger many of the changes in the biofilm through gradients of inter cellular activation. QS is triggered when the autoinducer concentration reaches a critical threshold because of cell density (Antunes and Ferreira 2009; Elias and Banin 2012). It functions as a feed-forward mechanism: the bond between signalling molecules and their bacterial receptors activates the expression of several genes, including those involved in the synthesis of these same signal molecules (Saxena et al. 2018). QS plays a pivotal role in the co-aggregation of different species of bacteria in multispecies biofilm ${ }^{8}$, in the increase of biomass during the formation of a monolayer and a multilayer structure, and it activates a large number of genes involved in the synthesis of matrix components (Karatan and Watnik 2009). Finally, many bacterial species employ QS to coordinate the disassembly of the EPS matrix by promoting the inhibition of matrix components synthesis, the degradation of the matrix, and the synthesis of surfactants (Solano et al. 2014).

The EPS matrix is a dynamic structure that consists of a variety of molecules (i.e. polysaccharides, proteins, lipids, extracellular DNA (eDNA), metal ions and water), which are bound together by weak physicochemical interactions (Flemming and Wingender 2010). The many functions of the EPS matrix from the retention of water to enzymatic activity, from the organisation of space to protective barrier - are at the origin of the common developmental dynamics, the metabolic co-dependence, and the enhanced

\footnotetext{
${ }^{6}$ Adhesins are cell-surface structures of bacteria that mediate transient or permanent surface attachment.

${ }^{7}$ Short range control relies on local cell-to-cell direct interactions. Medium range control is achieved when when an ensemble of cells is constrained for example by the ECM. In multicellular organisms such as animals it happens at the level of tissues. QS relies on signals and it can be considered as a distributed medium range control mechanism because it can affect a large number of cells by generating self-organised gradients. Long range control, instead, has a systemic reach and has the potential to constrain the activity of all the parts of the system. An example of long-range control mechanisms from animals is the release of hormones, distributed throughout the system through vascularization (see Bich et al. 2019 for more details).

${ }^{8}$ Let us consider, for example, the colonisation of the human oral cavity by the bacterial species Veillonella atypica and Streptococcus gordonii. In order to colonise dental surfaces, $V$. atypica requires the presence of $S$. gordonii, because $S$. gordonii ferments sugars and releases lactic acid, which constitutes the preferred carbon source for V. atypica. The coaggregation of bacteria from the two species is made possible by the fact that $V$. atypica produces a soluble chemical signal that triggers amylase expression in $S$. gordonii, thereby increasing the degradation of complex carbohydrates and lactic-acid production (Keller and Surette 2006).
} 
immunological response of biofilms. Through mechanical forces and concentrations of eDNA, extracellular signals and enzymes, the EPS matrix places several functional constraints on the cells of the biofilm and it actively contributes to the realisation and functioning of the overall organisation of the system (Bich et al. 2019). It makes the association of cells much more cohesive and coordinated than in the planktonic state, leading to a three-dimensional architecture (Steinberg and Kolodkin-Gal 2015). During biofilm development, the presence of the EPS matrix mechanically inhibits the rotation of the flagella of the cells and triggers intracellular signal cascades that increase the production and deposition of matrix molecules (Cairns et al. 2014). The 'activated matrix' (Flemming et al. 2007) - characterised by the presence of digestive enzymes, signal molecules, eDNA, lytic enzymes, etc. - is involved in the exchange of genetic material, in the control of cell behaviour, in the differentiation of cells into persister cells, spores, protease cells (Cairns et al. 2014; Steinberg and Kolodkin-Gal 2015), and in the control of the mobility of bacteria (Steinberg and KolodkinGal 2015). The EPS matrix promotes also the spatial proximity of cells and it is responsible for the presence of extracellular enzymes that give rise to an external digestive system, thus favouring integrated cometabolism ${ }^{9}$ and synthrophy ${ }^{10}$ among symbiotic partners (Dragoš and Kovács 2017). Moreover, fluids can flow throughout the biofilm by virtue of channels, realised by the EPS matrix, that allow the diffusion of nutrients to the cells of the innermost layers, and the distribution and removal of metabolic products (Sutherland 2001), also enabling medium range interaction and communication within the system.

The EPS matrix allows for the formation of different biochemical environments in such a way that otherwise incompatible bacteria (e.g. aerobic and anaerobic) may co-exist in the same biofilm. EPS matrix also reduces the diffusion rates of the compounds within the biofilm matrix itself, modulates gene expression patterns and decreases growth rates of the biofilm cells, making the biofilm robust with respect to external sources of perturbations and pathogens. In addition, the EPS matrix allows for the interconnection of innate and induced resistance factors that make the overall biofilm more resistant to external agents (Andersson and O'Toole 2008), and it favours multicellular strategies and a multilayer structure that inhibit the diffusion of antimicrobial agents within the biofilm (Stewart and Costerton 2001). As a result, biofilms achieve a certain form of collective immunological capability and gain a fitness advantage over their planktonic state (Burmølle et al. 2014).

In nature, multispecies biofilms tend to be more common than single-species biofilms. However, these latter are present in a variety of infections and on the surface of medical implants (O'Toole et al. 2000). Single- and multispecies biofilms are essentially similar both in the stages of the extracellular matrix deposition and degradation (attachment, maturation, and dispersion) and in the mechanisms involved in bacterial communication (quorum sensing) and bacterial conjugation. Nevertheless, they exhibit some differences in the interactions among bacterial partners. Multispecies biofilms exhibit much more variety of exchanges of nutrients and electrons than single-species biofilms, and thus they "gain energy from a series of reactions that a single species might lack" (Lohse et al. 2018, p. 27). Furthermore, it has been observed that the inclusion of other bacterial species in a single-species community may provide their members with numerous physiological advantages (e.g. passive resistance, metabolic cooperation, more efficient DNA sharing. See Wolcott et al. 2012). It is worth noting that, compared to single-species biofilms, multispecies biofilms can develop both cooperative relationships leading to increased biomass of the bacterial members and competitive relationships producing a decreased biomass of all members (Liu et al. 2016). Therefore, it seems that the life cycle of multispecies biofilms is subject to sharper fluctuations and, therefore, variability than that of single-species biofilms, thus potentially providing multispecies biofilms with increased capacities to invade surfaces, proliferate, and develop drug resistance. In the light of all these characteristics, it seems reasonable to suggest that both single- and multispecies biofilms exhibit the same kind of

\footnotetext{
9 By 'co-metabolism', we mean the simultaneous degradation of two compounds: the degradation of the second compound hinges on the presence of the first compound.

${ }^{10}$ By 'syntrophy', we refer to the phenomenon by which one species feeds on the by-products of another species.
} 
physiological integration enabled by the extracellular matrix; nonetheless, internal differentiation and functional diversity seems to be higher in some types of multispecies biofilms because higher number variety of metabolic exchanges between bacterial partners.

In the light of the above, what type (and degree) of integration - and therefore, of individuality - does this form of association achieve? Integration in biofilms is achieved by means of collective control exerted by QS mechanisms and EPS matrix at longer ranges than those that characterise basic cell-to-cell interactions alone. This is also a coarse-grained type control, based on differences in concentrations (of signals, control macromolecules, etc.), that is exerted gradually in space, at short and medium ranges, through gradients of signalling interactions (QS) and of distributions of EPS molecules. Although this form of control is not specific - i.e. does not rely on single interactions for a certain effect - it can give rise to a high variety of behaviours within the collective system.

From the organisational point of view, the EPS matrix structures are higher-level control subsystems (exerted over the individual cells) that contribute at medium ranges to the structural and functional cohesiveness and cooperation within biofilms. This is the reason why some authors have regarded biofilms as interactively and evolutionarily cohesive biological integrated individuals (Ereshefsky \& Pedroso, 2013, 2015; Doolittle, 2013), and even as full-fledged multicellular organisms (Shapiro 1988). However, due care should be exercised with regard to the type and degree of functional integration and individuality of biofilms, inasmuch as the capability of the EPS matrix to give rise to a fully integrated system is limited by several factors. Firstly, the EPS matrix does control the activity and fate of their cells only at short and medium ranges, but not at long ranges (relative to the scale of the system), due to the lack of full-fledged vascularisation, among other things (Bich et al. 2019). There is no specific constraint that has the capability of exerting a long-range type of control with a systemic reach. Global effects are achieved through selforganization, expanding usually by means of gradients, that is: they are the result of coarse-grained distributed control. Secondly, since the EPS matrix enables some degree of spatial segregation and functional differentiation only by means of gradients of concentrations, the internal modularity of the system is limited. Thirdly, the EPS matrix lacks components that make possible modularity and the construction of a global interface with the environment ${ }^{11}$.

In sum, while providing cohesiveness, the EPS does not establish clear-cut global boundaries or interfaces, nor long range control mechanisms. Therefore, if compared with specialised membrane mechanisms found in unicellular systems or interfaces such as the epithelium in eukaryotic multicellular systems, the EPS exerts a weaker and less specific control upon the permeability and selectivity of the system as a whole. Furthermore, the modulation of fluid transport by EPS channels is limited. This in turn limits the overall capability of the collective system to grow and to control cells at longer ranges.

Finally, as a consequence of the distinctive organisation realised by biofilms and the kind of control exerted within them, the type of (collective) reproduction carried out by biofilms is affected by the fact that the cells may keep their autonomy and revert cell-differentiation. Although some specialised cells may play the function of spores (e.g. in B. subtilis biofilm, Claessen et al. 2014) and some cheats (e.g. in P. aeruginosa biofilm) are considered as primitive forms of germ cells (Rainey and Kerr 2010; Hammerschmidt et al. 2014), this type of differentiation - which is the result of self-organisation starting from local interactions rather than specific control mechanisms - does not satisfy the requirements for units of selection. Moreover, each germ cell has its own history of mutation as a soma cell before randomly differentiating into a reproductive spore. In addition, most biofilms are characterised by the entrance and dispersion of cells, so that it can be claimed that biofilms do not exhibit a type of reproduction coordinated at the level of the whole system. The lack of a unified reproduction is even more evident in multispecies biofilms, where different genetic pools are represented in the absence of a reproductive bottleneck.

\footnotetext{
${ }^{11}$ For example, it lacks collagen IV, which promotes the realisation of interfaces and organ formation in eukaryotic multicellular systems, due to the role it plays in the basement membranes (Fidler et al. 2017).
} 
The fact that biofilms lack standard reproductive criteria for individuality (e.g. high levels of germ-soma specialization, unified reproductive lineages, reproductive bottlenecks) poses some challenging questions about whether or not they can be regarded as units of selection, and therefore evolutionary individuals. This issue forms the core of the debate between Clarke's (2016) and Ereshefsky and Pedroso's accounts (2013, 2015). According to Ereshefsky and Pedroso (2013, 2015), the criterion of evolutionary individuality based on the transference of genes from parents to offspring (vertical transmission) within the same lineage is too narrow. As an alternative, they propose a more open-ended approach according to which the members of prokaryotic associations (e.g. the prokaryotes of multispecies biofilms and consortia) share genes responsible for the mechanisms of trait transmission and reproduction. Thus, they "achieve evolutionary individuality but do not transmit their traits through single-species lineages. [...] Trait transmission in such consortia is accomplished through both lateral and vertical gene transfer, and the reproduction (or production) of such consortia is typically accomplished by aggregation" (Ereshefsky and Pedroso 2015, p. 10131). By contrast, Clarke (2016), who defends a view of heritage and evolutionary individuality based on parent-offspring lineage, has argued that the EPS matrix cannot give rise to a common lineage, and therefore it is not possible to regard biofilms as units of selection, since heritable variation occurs at the level of the single bacterial components rather than at the level of the biofilm as a whole.

\section{Endosymbiosis in prokaryotes: engulfment and its implications}

In this section, we analyse a very different form of prokaryotic association, one based on (asymmetric) engulfment of a species of bacteria within another (i.e. endosymbiosis). We are interested in this form of association because it is presumably of the type that led to a paradigmatic case of highly functionally integrated system: the eukaryotic cell and its organelles of endosymbiotic origin. Nevertheless, it is essential to underline that the endosymbiotic events that led to the origin of mitochondria and plastids in the eukaryotic cell were extremely rare in the prokaryotic world (Lane 2005; Booth and Doolittle 2015), probably because of the difficulty of overcoming conflicts between two prokaryotes fostering an endosymbiotic relationship.

Indeed, only one case of evolutionary stable ${ }^{12}$ endosymbiotic relationship between two prokaryotes has been discovered so $\mathrm{far}^{13}$ : a $\gamma$-proteobacterium (Candidatus Moranella endobia) that lives inside a $\beta$ proteobacterium (Candidatus Tremblaya princeps). This association is very peculiar because it is not capable of an independent form of life; indeed, it exists only enclosed in specialised cells (the bacteriocytes) of a specific organ (the bacteriome) of the mealybug insects ${ }^{14}$. Phylogenetically, the symbionts entered the mealybug at different times, the Tremblaya first and the Moranella later, so that their endosymbiotic relationship originated within the insect. In this sense, this endosymbiotic association shares some organisational features with the prokaryotic endosymbionts of sap-feeding insects, although in these latter cases all the prokaryotic symbionts live in the cytoplasm of eukaryotic cells (McCutcheon and von Dohlen 2011; von Dohlen et al. 2001). Like many endosymbionts of sap-feeding insects (e.g. Hodgkinia, Carsonella,

12 By 'evolutionary stable relationship', we mean a relationship that persist across several generations and that undergoes natural selection as a whole.

${ }^{13}$ Intracellular bacteria have been identified in some blue-green algae of the species Pleurocapsa minor in the seventies (Wujeck, 1979), but the physiology of this association has not been investigated. Other cases of intracellular bacteria invading the periplasm (e.g. Bdellovibrio) or the cytoplasm (e.g. Daptobacter) of other bacteria have been found (Corsaro and Venditti 2006). However, these cases represent transient symbiotic relationships (i.e. parasites) that do not give rise to an evolutionary stable relationship.

${ }^{14}$ For the sake of our argument, we just focus on the endosymbiotic relationship between the two bacteria (Tremblaya and Moranella), leaving aside the functional contribution of the insect. Indeed, this paper studies the functional integration of associations of prokaryotes and not the functional interdependence between prokaryotes and (multicellular) eukaryotes. Therefore, for clarity, hereinafter we will use the term 'host' to refer to Tremblaya, whereas the term 'endosymbiont' refers to Moranella. We will use "mealybug cells" for those eukaryotic cells that contain the Tremblaya-Moranella association. 
Sulcia, etc.), both Tremblaya and Moranella have extremely reduced genomes that affect their metabolic (i.e. anabolic and catabolic pathways), genomic (i.e. DNA replication, transcription and translation), and regulatory functions (i.e. metabolic regulation and gene regulation). Moranella's genome, which is four times larger than Tremblaya's ${ }^{15}$, codes for RNA molecules and proteins that cannot be expressed by Tremblaya's genome; but Moranella's genome is far from being self-sufficient and the functioning of this organism depends, in turn, on some of the few gene products from Tremblaya.

In spite of these specificities and of the impossibility to consider the consortium as a living fossil of an earlier step in the eukaryogenesis, the Moranella-Tremblaya association deserves a careful analysis for our purposes, precisely because it shows the early-stage implications of engulfment among two types of prokaryotic cells. Thus, it can shed light on the possibilities opened by this relationship and on the organisational problems it needs to overcome in order to maintain viability. Let us examine how engulfment affects this symbiotic relationship.

Engulfment creates a situation that is very different from the one brought about by the EPS matrix in biofilms, because now the different metabolic organisations (of the host, Tremblaya, and the endosymbiont, Moranella) share a common selective control boundary, i.e. the Tremblaya's membrane, a global constraint which enables a systemic long range control on all parts, and determines the type and degree of physiological integration between them. Moreover, since one of the organisms is located within the cytoplasm of the other, global viability requires a different type of functional coordination. Like many endosymbiotic relationships, Tremblaya and Moranella exhibit metabolic complementation, since the symbiotic partners partially contribute to the same metabolic pathways. A good example is provided by the metabolism of carbohydrates. Tremblaya has the genes encoding for only two enzymes of the pentose phosphate pathways (transaldolase B and transketolase). The rest of the enzymes for the pentose phosphate pathways, glycolysis, the phosphotransferase system, and the pyruvate dehydrogenase complex are expressed by Moranella's genome (López-Madrigal et al. 2013a). Amino acid biosynthesis constitutes another clear example of metabolic complementation: Tremblaya contains genes encoding for ten essential amino acids, but none of the amino acid pathways is complete in either Tremblaya or Moranella, so that these pathways need to be complemented by a patchwork of metabolites and enzymes from both partners (McCutcheon and von Dohlen 2011). Other metabolic pathways are incomplete (e.g. the tricarboxylic acid cycle) or absent (e.g. the nucleotide synthesis de novo or the synthesis of vitamins and cofactors) in the consortium.

The functional interlocking exhibited by the Tremblaya-Moranella association, however, is much deeper than metabolic complementarity and complementation - i.e., respectively, the exchange of metabolites or intermediate substrates - which are widespread in nature and characterise biofilms as well. Importantly, Tremblaya and Moranella jointly realise the [Fe-S] cluster ${ }^{16}$, which is usually not fully preserved in endosymbionts with reduced genomes (López-Madrigal et al. 2013a). The synthesis and assembly of this cluster requires a complex molecular machinery, and both members of the consortium are involved in the synthesis and maintenance of it, thus exhibiting a high degree of coordination. Another important aspect is that Tremblaya is totally dependent on Moranella for ATP synthesis, a feature that probably makes this consortium, according to Lopez-Madrigal et al. (2013a), the only known case in which all energy sources appear to be provided by only one of the partners. This is somehow analogous to what happens in the eukaryotic cell, where the mitochondria perform this function. Additionally, the cell-envelope structure is

\footnotetext{
15 Tremblaya's genome is 138,927 bp in length, whereas Moranella's is 538, 924 bp (McCutcheon and von Dohlen 2011). The difference in genome size between Tremblaya and Moranella is consistent with the hypothesis that Moranella penetrated Tremblaya as a secondary endosymbiotic event (López-Madrigal et al. 2013a).

${ }^{16}$ The [Fe-S]-cluster is a prosthetic group mainly involved in oxidation-reduction reactions. It plays several important functions related to energy metabolism and regulation. In particular, it plays a role in bacterial (and mitochondrial) respiratory complexes, in enzyme catalysis and in the sensing environmental or intracellular conditions to regulate gene expression (Lill 2009).
} 
simplified in both bacteria, because both Tremblaya and Moranella have lost most of the genes for the synthesis of murein and lipopolysaccharides.

The high degree of functional coordination between the endosymbiont and the host can be seen in the entangled way their genetic functions are realised ${ }^{17}$. Transcription requires the contribution of both organisms. Tremblaya encodes all the essential subunits of RNA polymerase and a single sigma factor, but it lacks the genes responsible for the basic transcription machinery, and for RNA processing and degradation. By contrast, Moranella has a minimal but complete transcription machinery and a number of genes encoding proteins that assist transcription. Furthermore, several transcriptional regulators, the functions of which are not yet fully known, and which are usually absent in endosymbionts with reduced genomes, have been retained by the genome of Moranella and they may play a role in the control of the transcription in this organism. Regarding translation, the consortium performs a very complex functional complementation ${ }^{18}$ and, according to López-Madrigal et al. (2013a), it may constitute the only known case for this specific function. While Moranella encodes more than $80 \%$ of the tRNA genes for the consortium, Tremblaya has retained tRNA genes for the most frequently used codons for alanine and, importantly, those for lysine, which are missing from Moranella's genome. Both Tremblaya and Moranella code for a high number of ribosomal proteins, giving rise to a ribosomal redundancy that could play a (not yet known) functional role for both symbiotic partners. However, only Moranella encodes ribosome maturation proteins and translational factors. In sum, the consortium shows a high degree of genetic complementarity: Tremblaya has lost most of the genes not only for metabolic but also for genomic functions, whereas Moranella has retained different genes for metabolism and genomic functions that complement those of Tremblaya, giving rise to a highly codependent relationship in which each partner partly contributes to the control mechanisms of the consortium.

In the light of the above, a fundamental question arises: how can this high degree of functional complementation happen? In order to answer to this question, we have to examine how Tremblaya and Moranella share functional constituents such as proteins through Moranella's membrane. In order to perform its essential cellular functions, Tremblaya needs to import from Moranella's cytoplasm not only metabolites, amino acids or carbon sources - as it is typical in common cases of endosymbiosis - but also functional control components such as proteins, tRNAs, ATP and molecular complexes (McCutcheon and von Dohlen 2011; López-Madrigal et al. 2011; López-Madrigal et al. 2013a) ${ }^{19}$. The consortium, therefore, requires a special transport system for the exchange of big molecules between the two partners ${ }^{20}$. Moranella's genome encodes a limited set of active transporters (e.g. the phosphotransferase system for the transport of hexoses) and two channels (MscL and YbaL) associated with osmotic stress, which play an important role in the excretion of low molecular weight molecules (e.g. ions and metabolites) and small cytoplasmic proteins. The Sec translocon ${ }^{21}$ machinery of Moranella exhibits a very reduced protein permeability and, therefore, it does not seem to be responsible for the provision of proteins and RNAs to Tremblaya. Hence, it has been hypothesised that the protein translocation from Moranella to Tremblaya may be due to a very primitive mechanism, yet effective in this case. It would consist in a transient perforation of the Moranella plasma

\footnotetext{
${ }^{17}$ See López-Madrigal et al. (2013a) for the details.

${ }^{18}$ By 'functional complementation', we mean the exchange of components that perform, or contribute to, specific functions (such as proteins, tRNA, parts of ribosomal machinery etc.) between the members of the association. It is different from 'metabolic complementation' which, instead, consists in the exchange of intermediate metabolic substrates.

${ }^{19}$ The same happens, in lesser degree, in the opposite direction from Tremblaya to Moranella.

${ }^{20}$ Exchanging small molecules such as amino acids and metabolites is deeply different than exchanging proteins, tRNA or other big molecules, and the two cases depend on distinct mechanisms of transport. Unlike our case study, the endosymbiotic relationship between an eukaryotic host and prokaryotic endosymbionts usually relies only on the exchange of amino acids between the two partners. The import of amino acids into the endosymbionts and the export of other amino acids to the host is usually mediated by transporters provided by the host (Duncan et al. 2014).

21 A translocon is a complex of secretory ('sec') proteins involved in the translocation of polypeptides across the membranes.
} 
membrane and of the osmotic channel MscL, controlled by osmotic stress ${ }^{22}$. It is made possible by two factors: 1) the peculiar composition of Moranella's membrane, more subject to perforation; and 2) the unequal distribution of metabolic products in the two partners. In this way, the cell wall of Moranella is transiently damaged and proteins would be able to reach Tremblaya cytoplasm (López-Madrigal et al. 2013b), thus allowing the two partners to exchange control components such as proteins and operate in an integrated way.

From the above, we can make some important remarks. The establishment of an endosymbiotic relationship between two bacteria is a condition that has rarely been observed in the biological world, because engulfment creates an intimate relationship within the cytoplasm of one of the partners which is difficult to maintain. Indeed, the absence of a compartmentalised nucleus makes the genome of the prokaryotic host, which is not protected by the nucleus membrane, more susceptible to a bombardment from pieces of DNA of endosymbiotic origin, in such a way that the host would be genetically unstable and, therefore, not adaptive (Lane 2015). This is one of the reasons why bacterial engulfment usually takes the form of a transient and ephemeral parasitic relationship, which ends when the prey (the "host") is killed (e.g. Vampirococcus and Bdellovibrio) or the predator leaves the prey (e.g. Daptobacter).

The endosymbiotic relationship between Tremblaya and Moranella shows the exceptional conditions required nowadays for a viable mutualistic relation. The massive loss of genes ${ }^{23}$, as demonstrated by Tremblaya's genome, implies that many metabolic pathways are absent or incomplete (e.g. those for amino acid biosynthesis). More importantly, some genetic functions (e.g. DNA replication, recombination, repair, and transcription), and regulatory processes (e.g. transcriptional regulators and translational factors) are severally undermined. As we have seen, most of these functions are either complemented or supplied by Moranella and thus require the joint action of both partners, thus suggesting a highly functional interconnected organisation in which each bacterium requires the other one. For these reasons, LopezMadrigal et al. $(2011$; 2013a) make the strong claim that " Ca. Tremblaya princeps' cannot be considered an independent organism, but that the consortium with its gammaproteobacterial symbiotic associate represents a new composite living being" (Lopez-Madrigal et al. 2011, p. 5587).

This case study is not aimed at putting the consortium in the same category as, for example, a eukaryotic cell, characterised by a number of specialised mechanisms that control the interaction between the cell and its organelles of symbiotic origin. We rather suggest that the engulfment between prokaryotes opens a series of challenges that need to be overcome in order to maintain a viable association. Unlike in bacterial biofilms, the establishment of a common boundary (the membrane of Tremblaya) makes possible the control of the global boundary conditions of the Tremblaya-Moranella system. While in biofilms the EPS matrix allows medium range control upon ensembles of cells, the endosymbiosis realized by the Tremblaya-Moranella association depends on a global spatial constraint (the membrane of Tremblaya), which has a (long-range) systemic reach upon all the components (the molecules and the endosymbionts in the cytoplasm of Tremblaya). Another important type of spatial constraint is the membrane of the Moranella endosymbionts. The presence of an internal spatial constraint, the membrane of Moranella symbionts, allows for a further

\footnotetext{
${ }^{22}$ Osmotic stress (or shock) is a sudden change in the solute concentration around a cell causing a change in the movement of water across the cell membrane.

${ }^{23}$ The loss of genes is an interesting feature of many commensal and mutualistic (symbiotic) relationships and it has been hypothesised that it increases the fitness of the overall associations. Morris et al. (2012) have coined the expression of "Black Queen hypothesis" to posit that "certain genes or, more broadly, biological functions, are analogous to the queen of spades. Such functions are costly and therefore undesirable, leading to a selective advantage for organisms that stop performing them. At the same time, the function must provide an indispensable public good, necessitating its retention by at least a subset of the individuals in the community (Morris et al. 2012). In most cases what is shared is metabolic products, giving rise to forms of 'syntrophic integration' by forming ecological networks (Skillings, 2019). In other cases the members of the association share functional components (under collective constraints such as EPS matrix or a common boundary), thus giving rise to forms of cross-control allowing for forms of 'physiological integration' (Bich, 2019).
} 
compartmentalization and modularity with potential for the evolution of specific controllers capable to modulate the permeability of the internal compartment, like it has happened in the case of organelles during eukaryogenesis. In this context, while exerting a systemic constraint at the global level, the endosymbiotic association exhibits more specific forms of cross control between the members, not achieved by means of self-organization only, but exerted by functional components from either Tremblaya or Moranella, or by functional components which are assembled from parts synthesized by both symbionts.

Moreover, the engulfment favours the genetic reduction and functional reorganisation of the two symbionts, thus leading to symbiotic partners that are necessarily less autonomous than those of a biofilm. A fundamental aspect of the integration between Tremblaya and Moranella is that they do not only exchange metabolites and amino acids, but also their main functional components. In the stable context of a nested endosymbiosis within the mealybug, this consortium has provided primitive, yet effective responses to the aforementioned challenges, such as the presumed passage of proteins, tRNA, etc. through mechanisms related to osmotic stress.

\section{The organisational implications of the EPS matrix and engulfment: a comparative discussion}

The previous two sections have highlighted the distinctive organisational features of biofilms and of the endosymbiosis between bacteria: their different types of collective borders which spatially constrain at different ranges the members of these associations and the forms of controls they enable, from coarsegrained distributed control in biofilms to more specific fine-grained forms of cross control among the partners of the endosymbiotic association. In this section, we compare their systemic implications. On the basis of this comparative discussion, we investigate the conceptual links between the engulfment of the Tremblaya-Moranella association and that of mitochondria and chloroplasts in the eukaryotic cell.

In the case of biofilms, the role of the collective spatial constraint is played by the EPS matrix: a dynamic extracellular structure that provides global cohesion, controls the activity of whole groups of bacteria (and archaea) at short and medium ranges, and maintains the cells adjacent to one another while differentiating gradients of space characterised by different boundary conditions, thus enabling functional differentiation and co-metabolism, syntrophy, common development and an enhanced immune response of the overall biofilm. In the case of the endosymbiosis between two species of bacteria, instead, engulfment provides a common global membrane (i.e. the membrane of Tremblaya). It is a global spatial constraint that favours an asymmetric relation between the host and the endosymbiont with a much more intimate and demanding collaboration between the members of the association, which allows for the establishment of systemic long range control (relative to the size of the whole system), and enables the realisation of fine-grained specific control mechanisms to coordinate the activities of the members.

The EPS matrix of biofilms and the membranes of the endosymbiotic associations impose on the symbiotic partners different types of spatial constraints that affect their collective physiological functions. Although both EPS matrix and engulfment allow metabolic coordination, they lead to different forms of collective metabolic organisations. In the case of biofilms, the spatial proximity of bacteria within the EPS matrix favours co-metabolism and synthrophy, and the release of enzymes in the EPS gives rise to an external digestive system. However, bacteria keep their autonomy and can in principle develop new metabolic relationships or leave the biofilm altogether. In the case of endosymbiosis, by contrast, engulfment implies a very specific set of selective pressures, that paves the way for a symbiotic "rabbit hole" in which incomplete metabolic pathways of the host are complemented by those of the endosymbiont and vice versa (Bennet and Moran 2015). Accordingly, in this inescapable association, metabolic interdependences are not facultative nor easily realisable by interchangeable partners, and they are also much more stable across time (i.e. over generations) if compared to the case of biofilms.

Yet the difference between bacterial endosymbiosis and biofilm is not limited to the stability of metabolic interdependencies, but includes two other aspects that have deeper organisational implications. In the first 
place, the bacterial EPS represents a collective and fuzzy spatial constraint, with little and unspecific control upon the passage of molecules or organisms through it. In the case of engulfment, instead, the membrane of the host provides the system with a common selective border characterised by global and more precise mechanisms of control of permeability and transport (i.e. the capability to modulate the internal $\mathrm{pH}$, concentrations of metabolites, osmotic pressure, spatial and temporal distribution of specific chemicals). With regard to the internal organisation of space, the EPS allows for a coarse-grained spatial differentiation, while the membrane of the endosymbionts provides the endosymbiotic association with much more modularity due to the presence of internal compartments, opening up the possibility for a fine-grained control of permeability of the endosymbiont membrane and targeted transport of proteins.

In the second place, fundamental differences between the two types of associations arise also in the ways they control intercellular relations. In biofilms, QS and the EPS matrix exert a distributed control upon the cells at short and medium ranges, by realising gradients of signalling interactions (QS) and of distributions of EPS molecules. In engulfed symbiosis, instead, functional coordination requires specific control at all ranges and avoidance of conflict. In the case of the Moranella-Tremblaya association, for instance, proteins, tRNA and other control molecules of Moranella need to pass across its membrane to enter the cytoplasm of Tremblaya (where they can directly control different biosynthetic processes or regulate basic functions). To a lesser degree, the same happens to some control molecules from Tremblaya, so that the whole consortium maintains viability through a very basic form of cross-control ${ }^{24}$ and interlocked regulation ${ }^{25}$. Thus, engulfment cannot succeed unless a tight control of the most fundamental functions of both the host and the endosymbiont is established. This requires a deep functional re-organisation with an irreversible loss of autonomy of the former partners.

For these reasons, we suggest that the engulfment between two prokaryotes constitutes the fundamental requirement for the appearance of a strong, and non-facultative, functional integration between different symbiotic partners. This type of relationship is very demanding in organisational terms, insofar as such a specific control requires: (1) the presence of the right components in the right place at a given time; and (2) the implementation of mechanisms for transporting proteins and other complex control macromolecules across the membrane of the endosymbiont, not only basic building blocks such as metabolites and amino acids $^{26}$.

These different types of physiological integration pose some difficult questions about the relationship between functional integration, system-level coordinated reproduction, and heredity. It is not our purpose to find a solution to this complex issue. However, we suggest that in (prokaryotic) collective organisations a certain level of physiological integration is required to gain the capability of reproducing as a unit, because the parts need to be functionally differentiated (e.g. between germ and soma) and their activities coordinated for this purpose. For example, under starvation conditions, some single-species biofilms (e.g. B. subtilis or M. xanthus) can produce collective forms of reproduction (e.g. the spores of B. subtilis or the fruiting bodies of M. xanthus) by means of (local) contact mediated signals, that are enabled by the spatial proximity of cells, and the resulting formation of gradients through self-organisation (Julien et al. 2000; Muñoz-Dorado et al. 2016). Thus, the existence of an extracellular matrix, which keeps bacteria close to one another, may play an organizational role not only in establishing a certain kind of physiological integration, but also a diffused

\footnotetext{
${ }^{24}$ By 'cross-control', we mean one partner producing the components that control processes in the other.

${ }^{25}$ By 'interlocked' regulation, we mean the activity of regulatory mechanisms which rely on the components produced by both partners.

${ }^{26}$ The Moranella-Tremblaya consortium realises transport through a very basic mechanism based on osmosis in presence of a weakened membrane. In spite of being unspecific and inefficient, it can guarantee the viability of the consortium in the very stable environment of mealybug cells. In fact, this particular mechanism lacks complex channels and mechanisms for protein targeting that would allow much more specific control upon the localisation of functional components. A more stable and robust solution to this problem would instead require a much deeper re-organisation of the systems involved, which is indeed what it is supposed to have happened during the process of eukaryogenesis.
} 
and transient, context-dependent (i.e. dependent on starvation conditions) collective reproductive system. This reproductive system is realised through coarse-grained control, based on local interactions and the resulting formation of gradients, rather than coordinated by means of global specific control mechanisms. Similarly, some 'multi-cellular prokaryotes' (Claessen et al. 2014; Lyons and Kolter 2015) such as filamentous bacteria (e.g. N. punctiforme), actinomycetes (e.g. A. Israeli) or beggiatoa (e.g. B. leptomitoformis) exhibit distinct kinds of collective reproduction that seem to be enabled by their spatial contiguity (e.g. through proteinaceous complexes) and a minimal degree of functional integration (e.g. through intercellular signals, metabolic co-dependence. See Claessen et al. 2014). In the case of endosymbiosis, engulfed symbionts are so tightly integrated that they cannot survive autonomously, and the endosymbionts can only be transmitted vertically due to the role of the host's membrane as global constraint. Consequently, the genes of both the host and the endosymbionts jointly change, and these variations can be selectively transmitted to the new generations, making the whole system a unit of selection. Moreover, engulfment allows for the implementation of further fine-tuned regulatory mechanisms during the evolution of the symbiosis to synchronise the processes of growth and division more precisely.

A basic form of coarse-grained physiological integration is therefore a necessary, but not sufficient, condition for a collective reproduction and a vertical transmission of genes, as shown by biofilms. Although they have an EPS matrix that allows distributed mechanisms of regulatory control and signalling for synchronising the members of the association, neither a global (long-range) control upon the reproduction of the components, nor unified mechanisms for the differential variation of the gene pool of a biofilm have been reported in the current literature so far (see, for example, Lopez et al. 2009; Liu et al. 2016). As a result, the cells of single and multispecies biofilms may evolve independently from one another rather than undergo coselection, as evolutionary individuals instead do. By contrast, the type of integration enabled by engulfment, as a common boundary and control constraint, paves the way not only for a higher degree of integration and functional differentiation, but also for a collective reproduction, despite the fact that the species involved are different.

The engulfed association among prokaryotes imposes a specific set of constraints on the symbiotic partners, which determine a dramatic reduction in the endosymbiont genome, and lead to an irreversible functional dependence between the symbiotic partners. Despite its limits, the relevance of the relationship between Tremblaya and Moranella consists in the fact that it involves not only a sophisticated complementation of metabolic and genomic functions between the host and the endosymbiont, but also a control upon the localisation of proteins and the reproduction and development of the endosymbionts. These dynamics, which are hardly sketched in the consortium Tremblaya-Moranella, achieve the highest expression in the eukaryotic organelles of endosymbiotic origin, such as mitochondria and chloroplasts, to give rise to full-fledged functionally integrated systems: the eukaryotic cells. Since most of the proteins controlling and regulating the internal processes of proto-mitochondria and proto-chloroplasts were progressively encoded in the nucleus and synthesised in the cytoplasm of the (host) cell, the appearance, among other things, of a protein import and targeting machinery played a major role in the conversion of endosymbionts into organelles. It allowed the host to directly control and regulate the functions of the future organelle and of the overall consortium (Martin 2010; Cavalier-Smith 2007).

In sum, a viable engulfment establishes several constraints on the organisations of both the host and the endosymbionts that are much tighter and demanding than those placed by the EPS matrix on the bacteria of a biofilm. It is not a coincidence, therefore, that only very few cases of prokaryotic endosymbiosis have been discovered so far. The evolutionary stable internalisation of a prokaryotic organism within another one raises a series of issues whose solution leads to a deeper reproductive, developmental and metabolic integration, based on a more precise form of systemic control. Ultimately, they may give rise to the appearance of a strong integrated identity. The EPS matrix, instead, leads to more ephemeral, although highly successful, organisations, in which symbiotic partners retain a basic autonomy and are kept together by distributed forms of control, without a fine-tuned control of the overall development and reproduction. 


\section{Concluding remarks}

Our case study shows that in the prokaryotic world, a process of association of individuals may lead to different forms of collective units, with different types and degrees of integration. In this context, functional integration can be broadly understood as a phenomenon that originates when a set of different and initially autonomous organisations (each one with its own functional parts) begins to functionally cooperate and share their local functions. It leads to the establishment of a wider collective organisation where some functional constraints of the constituent organisations are interlocked and control one another's processes in such a way that the whole system achieves viability. In this context we have identified some crucial elements that enable different types and degrees of functional integration, specifically: (1) different types and ranges of collective spatial constraints exerted by the EPS in biofilms and by the global membrane in the endosymbiotic association; (2) different forms of control exerted by both the spatial and the intercellular control mechanisms, i.e. the distributed coarse grained control characteristic of biofilms and the specific fine-tuned control realised in the case of endosymbiosis; and (3) the different degrees of cross-control and interlocked regulation that are required in order to modulate and coordinate intercellular interactions in the different associations, with especially strong requirements for the endosymbiotic case of Tremblaya and Moranella, where many of these mechanisms are assembled from functional components produced by both partners.

However, the necessity of the associated parts to achieve a global viability implies that there could not be an indefinite number of integration possibilities. On the contrary, given a specific set of entities, only a discrete number of collective organisations are stable on the physiological and evolutionary scales. In our case, we can find either biofilm-type forms of association or endosymbiotic associations of prokaryotic cells; but the latter seem, for all the reasons that we have analysed, quite rare and fragile until a completely new organisation - the eukaryotic cell - is realised.

As we have seen, the appearance of a strong form of functional integration in the prokaryotic domain is a process characterised by an initial step where different individualities (autonomous entities) enter in a process of irreversible association. Most symbiotic associations of prokaryotes, like biofilms, cannot be considered as full-fledged functionally integrated individuals, but rather as communities of (sometimes highly) coordinated organisms, kept together by means of distributed control mechanisms. Among these associations, the key for the achievement of a strong functional integration is the creation of an asymmetric compartmentalisation: a spatial border, a global constraint that functionally acts as a selective frontier between the associated system and the external environment. At the same time, engulfment is a much more difficult way of achieving a stable association, and it is at the origin of a long evolutionary travel full of conflicts.

Engulfment triggers a cascade of events that opens up (and forces) several possibilities for structural and functional reorganisation and biological novelty in both symbiotic partners. Although we have no traces or examples of the presumably long process that led to the eukaryotic cell, our case study suggests that this process might have likely involved the modification or loss of old functions and the appearance of new capacities, reaching more or less viable intermediate stages until a global robust viability was reached. Viable internalisation could only have been achieved and maintained by increasing functional integration through new forms of global control, starting from the modulation of the permeability of the common boundary, to different systems of transport and targeting of functional components between the partners through the endosymbionts' membranes, which in turn made possible the implementation of precise mechanisms of cross-control and interlocked regulation. All of them contributed towards the generation of a new and stronger form of individuality with a regulatory machinery in charge of all the internal functions, exemplified by the composite organisation of a eukaryotic cell, where the original endosymbiotic cells lost their former autonomy and became organelles. The emergence of a new functionally integrated organisation, 
therefore, requires a functional redefinition of both the original organisms and of the symbiotic consortium as a whole

In sum, functional integration can generally be defined as the degree to which the different components of a biological dynamic regime of self-maintenance depend on one another for their production, maintenance, activity and reproduction. If we take the eukaryotic cell as the reference example of new forms of fullfledged biological individuality resulting from association between prokaryotes, individuality can be understood in terms of the degree, scale and precision of the control and coordination of the parts that collectively make the system a viable functional whole (i.e. an integrated unit). To do so, even the minimal forms of biological (and, likely, proto-biological) organisation require, in the first place, some internal functional differentiation (Mossio et al. 2009). A cohesive integration between different functional tasks is achieved, then, when the differentiation of functions is coordinated at the system level by control and regulatory mechanisms that (1) act across the different entities participating in the association, and (2) are exerted in such a way that the differentiated components can contribute through their activity to the maintenance of the system. As we have seen, biological systems can give rise to different forms of functional associations, exhibiting different degrees of integration. In this process of integration, the deeper the codependency between the original organisations, the higher is their progressive loss of autonomy, accompanied by the appearance of new forms of control upon the members of the association.

\section{Acknowledgements}

The authors acknowledge funding from the Basque Government (Project: IT1228-19), Ministerio de Ciencia, Innovación y Universidades, Spain (research project PID2019-104576GB-I00 for GM, LB and AM, and 'Ramon y Cajal' Programme RYC-2016-19798 for LB), University of the Basque Country, Spain (predoctoral scholarship PIF17/31 for GM).

\section{References}

Andersson GG, O'Toole GA (2008) Innate and induced resistance mechanisms of bacterial biofilms. In: Romeo T (ed) Bacterial Biofilms. Springer, Berlin Heidelberg, pp 85-105. https://doi.org/10.1007/9783-540-75418-3_5

Antunes LC, Ferreira RB (2009) Intercellular communication in bacteria. Crit Rev Microbiol 35:69-80. https://doi.org/10.1080/10408410902733946

Arnellos A, Moreno A, Ruiz-Mirazo K (2014) Organisational requirements for multicellular autonomy: insights from a comparative case study. Biol Philos 29:851-884. https://doi.org/10.1007/s10539-0139387-x

Bennett GM, Moran NA (2015) Heritable symbiosis: The advantages and perils of an evolutionary rabbit hole. Proc Natl Acad Sci U S A 112:10169-76. https://doi.org/10.1073/pnas.1421388112

Bich L (2019). The problem of functional boundaries in prebiotic and inter-biological systems. In: Minati, G, Pessa E, Abram, M (eds) Systemics of Incompleteness and Quasi-Systems. Springer, New York, pp 295-302. https://doi.org/10.1007/978-3-030-15277-2_23

Bich L, Mossio M, Ruiz-Mirazo K, Moreno A (2016) Biological regulation: controlling the system from within. Biol Philos 31:237-265. https://doi.org/10.1007/s10539-015-9497-8

Bich L, Pradeu T, Moreau JF. (2019) Understanding Multicellularity: The Functional Organization of the Intercellular Space. Front Physiol 10:1170. https://doi.org/10.3389/fphys.2019.01170 
Booth A, Doolittle WF (2015) Eukaryogenesis, how special really?. PNAS 112:10278-10285. https://doi.org/10.1073/pnas.1421376112

Burmølle M, Ren D, Bjarnsholt T, Sørensen SJ (2014) Interactions in multispecies biofilms: do they actually matter? Trends Microbiol 22:84-91. https://doi.org/10.1016/j.tim.2013.12.004

Cairns LS, Hobley L, Stanley-Wall, NR (2014) Biofilm formation by Bacillus subtilis: new insights into regulatory strategies and assembly mechanisms. Mol Microbiol 93: 587-598. https://doi.org/10.1111/mmi.12697

Catania F, Krohs U, Chittò M, Ferro D, et al (2017) The hologenome concept: we need to incorporate function. Theory Biosci 136:89-98. https://doi.org/10.1007/s12064-016-0240-z

Cavalier-Smith T (2007) The Chimaeric Origin of Mitochondria: Photosynthetic Cell Enslavement, GeneTransfer Pressure, and Compartmentation Efficiency. In: Martin WF, Müller Miklós (eds) Origin of Mitochondria and Hydrogenosomes. Springer, Berlin Heidelberg, pp 160-199. https://doi.org/10.1007/978-3-540-38502-8_8

Claessen D, Rozen DE, Kuipers OP, Søgaard-Andersen L, van Wezel GP (2014) Bacterial Solutions to Multicellularity: A Tale of Biofilms, Filaments and Fruiting Bodies. Nat Rev Microbiol 12:115-124. https://doi.org/10.1038/nrmicro3178.

Clarke E (2016) Levels of selection in biofilms: multispecies biofilms are not evolutionary individuals. Biol Philos 31: 191-212. https://doi.org/10.1007/s10539-016-9517-3

Corsaro D, Venditti D (2006) Bacterial Endosymbionts in Prokaryotes. In: Shively JM (ed) Complex Intracellular Structures in Prokaryotes. Microbiology Monographs, vol 2. Springer, Berlin, Heidelberg, pp 359-371. https://doi.org/10.1007/7171_035

von Dohlen CD, Kohler S, Alsop ST, McManus WR (2001) Mealybug $\beta$-proteobacterial endosymbionts contain $\gamma$-proteobacterial symbionts. Nature 412: 433-436. https://doi.org/10.1038/35086563

Doolittle WF (2013) Microbial neopleomorphism. Biol Philos 28: 351-378. https://doi.org/10.1007/s10539012-9358-7

Dragoš A, Kovács ÁT (2017). The Peculiar Functions of the Bacterial Extracellular Matrix. Trends Microbiol 25:257-266. https://doi.org/10.1016/j.tim.2016.12.010

Duncan RP, Husnik F, Van Leuven JT, Gilbert DG, Dávalos LM, McCutcheon JP, Wilson ACC (2014) Dynamic recruitment of amino acid transporters to the insect/symbiont interface. Mol Ecol 23:16081623. https://doi.org/10.1111/mec. 12627

Dupré J, O’Malley MA (2009) Varieties of Living Things: Life At The Intersection Of Lineage And Metabolism. Philos Theor Biol 1:e003. http://dx.doi.org/10.3998/ptb.6959004.0001.003

Elias S, Banin E (2012) Multi-species biofilms: living with friendly neighbors. FEMS Microbiol Rev 36:990-1004. https://doi.org/10.1111/j.1574-6976.2012.00325.x

Ereshefsky M, Pedroso M (2013) Biological individuality: the case of biofilms. Biol Philos 28: 331-349. https://doi.org/10.1007/s10539-012-9340-4

Ereshefsky M, Pedroso M (2015) Rethinking evolutionary individuality. PNAS 112:10126-10132. https://doi.org/10.1073/pnas.1421377112 
Fidler AL, Darris CE, Chetyrkin SV, et al (2017) Collagen IV and basement membrane at the evolutionary dawn of metazoan tissues. eLife, 6. https://doi.org/10.7554/eLife.24176.001

Flemming HC, Neu TR, Ozniak DJ (2007) The EPS Matrix: The "House of Biofilm Cells". J Bacteriol 189:7945-7947. https://doi.org/10.1128/JB.00858-07

Flemming HC, Wingender $\mathbf{J}$ (2010) The biofilm matrix. Nat Rev Microbiol 8: 623-633. https://doi.org/10.1038/nrmicro2415

Griesemer J (2016) Reproduction in Complex Life Cycles: Toward a Developmental Reaction Norms Perspective. Philos Sci 83: 803-815. Doi: 10.1086/687865

Hammerschmidt K., Rose C., Kerr B, Rainey PB (2014) Life cycles, fitness decoupling and the evolution of multicellularity. Nature 515: 75-79. https://doi.org/10.1038/nature13884

Howes, M (1998). The Self of Philosophy and the Self of Immunology. Perspect. Biol. Med 42 (1):118-130.

Hull DL (1980) Individuality and Selection. Ann Rev Ecol Syst 11:311-332. https://doi.org/10.1146/annurev.es.11.110180.001523

Julien B., Kaiser D., Garza A. (2000). Spatial control of cell differentiation in Myxococcus xanthus. Proc. Natl. Acad. Sci. U.S.A. 97, 9098-9103. https://doi.org/10.1073/pnas.97.16.9098

Karatan E, Watnik P (2009) Signals, Regulatory networks, and Materials That Build and Break Bacterial Biofilms. Microbiol Mol Biol Rev 73:310-347. https://doi.org/10.1128/MMBR.00041-08

Keller L, Surette MG (2006) Communication in bacteria: an ecological and evolutionary perspective. Nat Rev Microbiol 4(4):249-258

Lane N (2005) Power, Sex, Suicide. Oxford University Press, Oxford

Lane N. (2015) The vital question. W W Norton Company, New York.

Lill $\mathrm{R}$ (2009) Function and biogenesis of iron-sulphur proteins. Nature 460:831-838. https://doi.org/10.1038/nature08301

Liu W, Røder HL,1 Madsen JS, Bjarnsholt T,3 Sørensen SJ,1 Burmølle M (2016) Interspecific Bacterial Interactions are Reflected in Multispecies Biofilm Spatial Organization. Front Microbiol. 7: 1366. https://doi.org/10.3389/fmicb.2016.01366

Lohse MB, Gulati M, Johnson AD, Nobile CJ (2018) Development and Regulation of Single- And MultiSpecies Candida Albicans Biofilms. Nat Rev Microbiol 16:19-31. https://doi.org/10.1038/nrmicro.2017.107

Lopez D, Vlamakis H, Kolter R (2009) Generation of multiple cell types in Bacillus subtilis. FEMS Microbiol Rev 33:152-163. https://doi.org/10.1111/j.1574-6976.2008.00148.x

López-Madrigal S, Balmand S, Latorre A, Heddi A, Moya A, Gil R (2013b) How does Tremblaya princeps get essential proteins from its nested partner Moranella endobia in the Mealybug Planoccocus citri?. PLoS One 8:e77307. https://journals.plos.org/plosone/article?id=10.1371/journal.pone.0077307

López-Madrigal S, Latorre A, Porcar M, Moya A, Gil R (2011) Complete genome sequence of "Candidatus Tremblaya princeps" strain PCVAL, an intriguing translational machine below the living-cell status. J Bacteriol 193: 5587-8. https://jb.asm.org/content/193/19/5587 
López-Madrigal S, Latorre A, Porcar M, Moya A, Gil R (2013a) Mealybugs nested endosymbiosis: going into the 'matryoshka' system in Planococcus citri in depth. BMC Microbiol 13: 74. https://doi.org/10.1186/1471-2180-13-74

Lyons NA, Kolter R (2015) On The Evolution of Bacterial Multicellularity. Curr Opin Microbiol. 2015 24: 21-28. https://doi.org/10.1016/j.mib.2014.12.007

Martin W (2010) Evolutionary origins of metabolic compartmentalisation in eukaryotes. Philos Trans R Soc Lond B Biol Sci 365:847-855. https://doi.org/10.1098/rstb.2009.0252

McCutcheon JP, von Dohlen CD (2011) An Interdependent Metabolic Patchwork in the Nested Symbiosis of mealybugs. Curr Biol 21:1366-1372. https://doi.org/10.1016/j.cub.2011.06.051

Moreno A, Mossio M (2015) Biological Autonomy: A Philosophical and Theoretical Enquiry. Springer, Dordrecht

Morris JJ, Lenski RE, Zinser ER (2012) The Black Queen Hypothesis: Evolution of Dependencies Through Adaptive Gene Loss. mBio 3:e00036-12. doi: 10.1128/mBio.00036-12

Mossio M, Saborido C, Moreno A (2009) An Organisational Account of Biological Functions. Brit J Phil Sci 60:813-841. https://doi.org/10.1093/bjps/axp036

Muñoz-Dorado J, Marcos-Torres FJ, García-Bravo E, Moraleda-Muñoz A, Perez J (2016) Myxobacteria: Moving, Killing, Feeding, and Surviving Together. Front Microbio 17:781. https://doi.org/10.3389/fmicb.2016.00781

O’Toole, G., Kaplan, H. B., \& Kolter, R. (2000). Biofilm Formation as Microbial Development. Ann Rev Microbiol, 54(1), 49-79. https://doi.org/10.1146/annurev.micro.54.1.49

Pradeu T (2010) What is An Organism? An Immunological Answer. Hist Phil Life Sci 32:247-268. https://doi.org/10.2307/23335074

Rainey PB, Kerr B (2010) Cheats as First Propagules: A New Hypothesis for the Evolution of Individuality During the Transition From Single Cells to Multicellularity. Bioessays 32:872-80. https://doi.org/10.1002/bies.201000039

Reyes-Prieto et al. (2014). Scanty microbes, the 'symbionelle' concept. Environ Microbiol.16(2):335-8. doi: $10.1111 / 1462-2920.12220$

Queller DC, Strassmann JE (2009) Beyond society: the evolution of organismality. Philos Trans R Soc Lond B Biol Sci 364:3143-3155. https://dx.doi.org/10.1098\%2Frstb.2009.0095

Queller DC, Strassmann JE (2016) Problems of multi-species organisms: endosymbionts to holobionts. Biol Philos 31:855-873. http://dx.doi.org/10.1007/s10539-016-9547-x

Saxena P, Joshi Y, Rawat K, Bishit R (2019) Biofilms: Architecture, Resistance, Quorum Sensing and Control Mechanisms. Indian J Microbiol 59:3-12. https://doi.org/10.1007/s12088-018-0757-6

Shapiro JA (1988) Bacteria as multicellular organisms. Oxford University Press, New York

Skillings DJ (2016) Holobionts and the Ecology of Organisms - Multi-Species Communities or Integrated Individuals?. Biol Philos 31:875-892. https://doi.org/10.1007/s10539-016-9544-0 
Skillings, D. (2019). Trojan Horses and Black Queens: 'causal core' explanations in microbiome research. Biol Philos, 34(6), 60. doi:10.1007/s10539-019-9718-7

Sober E (1991) Organisms, Individuals, and Units of Selection. In: Tauber AI (ed) Organisms and the Origins of Self. Kluwer, Dordrecht, pp 275-296

Solano C, Echeverz M, Lasa I (2014) Biofilm dispersion and quorum sensing. Curr Opin Microbiol 18:96104. https://doi.org/10.1016/j.mib.2014.02.008

Steinberg N, Kolodkin-Gal I (2015) The Matrix Reloaded: Probing the Extracellular Matrix Synchronizes Bacterial Communities. J Bacteriol 197:2092-2103. https://doi.org/10.1128/JB.02516-14

Stewart PS, Costerton JW (2001) Antibiotic resistance of bacteria in biofilms. Lancet 358:135-138. https://doi.org/10.1016/S0140-6736(01)05321-1

Sutherland IW (2001) The biofilm matrix--an immobilized but dynamic microbial environment. Trends Microbiol 9:222-227. https://doi.org/10.1016/S0966-842X(01)02012-1

Wilson EO (1974) The Perfect Societies. Science 184:54-55. https://doi.org/10.1126/science.184.4132.54

Wolcott R, Costerton JW, Raoult D, Cutler SJ (2012) The Polymicrobial Nature of Biofilm Infection. Clin Microbiol Infect 19:107-12. https://doi.org/10.1111/j.1469-0691.2012.04001.x

Wujek D.E (1979) Intracellular Bacteria in the Blue-Green Alga Pleurocapsa minor. T Am Microsc Soc 98:143. https://doi.org/10.2307/3225953 